\title{
MMSE Soft-Interference-Cancellation Aided Iterative Center-Shifting $K$-Best Sphere Detection for MIMO Channels
}

\author{
Li Wang, Lei Xu, Shen Chen and Lajos Hanzo \\ School of ECS, University of Southampton, SO17 1BJ, UK. \\ Tel: +44-23-8059 3125, Fax: +44-23-8059 4508

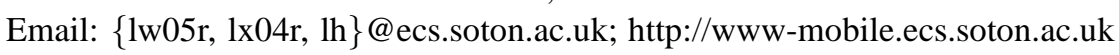

\begin{abstract}
Based on an EXtrinsic Information Transfer (EXIT) chart-assisted receiver design, a low-complexity near-Maximum A Posteriori (MAP) detector is constructed for high-throughput systems. A high throughput is achieved by invoking high-order modulation schemes or multiple transmit antennas, while employing a novel sphere detector (SD) termed as a center-shifting SD scheme. The center-shifting SD is assisted by the MMSE softinterference-cancellation (SIC-MMSE) algorithm. The resultant scheme is capable of attaining a considerable complexity reduction over the conventional SD-aided iterative benchmark receiver. For example, the SIC-MMSE center-shifting scheme may enable the iterative receiver to achieve a near-MAP performance in the challenging scenario of an $(8 \times 4)$-element rank-deficient 4-QAM SDM/OFDM system. This near-MAP performance is achieved, despite imposing a reduced detection-candidate-list-generationrelated complexity, which is about an order of magnitude lower than that exhibited by the list-SD dispensing with the proposed center-shifting scheme. As a further benefit, the computational complexity associated with the extrinsic LLR calculation was reduced by a factor of about 64 . The associated memory requirements were also reduced by a factor of 64 .
\end{abstract}

\section{INTRODUCTION}

In recent years the sphere detection (SD) techniques $[1,2]$, have received wide interest in both the research and industrial communities, which are capable of achieving near-Maximum A Posteriori (MAP) performance at a significantly lower computational complexity compared to the classic MAP detectors. According to the specific tree-search technique employed, SDs can be roughly classified into two types: the family of depth-first [1] and breadth-first SDs [2]. The $K$-best SD using breadth-first search has several advantages over its depth-first counterparts, such as for example imposing a fixed detection complexity and a flexible hardware implementation. However, it tends to suffer from a performance degradation unless $K$, namely the number of candidates retained at each tree search level, is sufficiently large especially in high-throughput systems either employing high-order modulation schemes or a large number of transmit antennas.

Acknowledgements: The work reported in this paper has formed part of the Core 4 Research Programme of the Virtual Centre of Excellence in Mobile and Personal Communications, Mobile VCE, www.mobilevce.com, whose funding support, including that of EPSRC, is gratefully acknowledged. Fully detailed technical reports on this research are available to Industrial Members of Mobile VCE.
Our main contribution in this paper is the design of a novel low-complexity near-MAP soft interference cancellation assisted center-shifting SD, which is capable of significantly improving the performance of the conventional SD-aided iterative receiver.

The rest of this paper is organised as follows. Section II describes the system model of our Multiple-Input MultipleOutput (MIMO) aided OFDM system. The center-shifting technique and the proposed receiver architecture are presented in Section III. In Section IV we provide our simulation results, while in Section V we provide our conclusions.

\section{System Model}

In this paper a spatial-division-multiplexing (SDM) orthogonal-frequency-division-multiplexing (OFDM) system equipped with $M$ transmit antennas and $N$ receiver antennas is considered [3]. The subcarrier-related MIMO-OFDM system model considered is given by $[3,4]$ :

$$
\mathbf{y}=\mathbf{H s}+\mathbf{w},
$$

where $\mathbf{y} \in \mathbb{C}^{N \times 1}, \mathbf{s} \in \mathbb{C}^{M \times 1}$ and $\mathbf{w} \in \mathbb{C}^{N \times 1}$ denote the received signal and transmitted signal vector as well as the AWGN sample vector, respectively. Moreover, $\mathbf{H}$ is a $(N \times M)$-dimensional frequency-domain channel transfer factor (FDCHTF) matrix with each column representing the unique spatial signature of the corresponding transmit antenna. Here, we assume that the FDCHTFs are independent, stationary, complex valued Gaussian distributed processes with a zero-mean and a unit variance [3]. Furthermore, both the transmitted signal of transmit antenna element $m, s^{(m)}$, and the AWGN noise, $w_{n}$, encountered at the $n$th receive antenna element exhibit a zero-mean and a variance of $\sigma_{s}^{2}$ and $\sigma_{w}^{2}$, respectively.

\section{SIC-MMSE-AIDED CENTER-SHIFTING SD}

\section{A. Principle of Sphere Detection}

1) Transformation of the ML Metric: The well-known ML solution is given by:

$$
\hat{\mathbf{s}}_{M L}=\arg \min _{\tilde{\mathbf{s}} \in M_{c}^{M}}\|\mathbf{y}-\mathbf{H} \check{\mathbf{s}}\|_{2}^{2},
$$

where $M_{c}$ is the number of modulated symbol points in the constellation and $M$ is the number of transmit antennas 
employed by the system. Observe from 2 that a potentially excessive-complexity search may be encountered, depending on the value of $M_{c}$ and/or $M$, which prevents the application of the full-search-based ML detectors in high-throughput scenarios. However, under the assumption of using constant modulus modulation schemes, such as BPSK and 4-QAM, and comparing the unconstrained MMSE solution of $\hat{\mathbf{x}}_{c}=$ $\left(\mathbf{H}^{H} \mathbf{H}+\sigma_{w}^{2} \mathbf{I}\right) \mathbf{H}^{H} \mathbf{y}$ to all legitimate constrained/sliced solution, the ML solution of Eq.(2) can be transformed into [5]:

$$
\hat{\mathbf{s}}_{M L}=\arg \min _{\check{\mathbf{s}} \in M_{c}^{M}}\left(\check{\mathbf{s}}-\hat{\mathbf{x}}_{c}\right)^{H}\left(\mathbf{H}^{H} \mathbf{H}+\sigma_{w}^{2} \mathbf{I}_{\mathcal{C}}\right)\left(\check{\mathbf{s}}-\hat{\mathbf{x}}_{c}\right) .
$$

2) Channel Matrix Triangularization: Let us obtain the $(M \times M)$ upper-triangular matrix $\mathbf{U}$, which satisfies $\mathbf{U}^{H} \mathbf{U}=\mathbf{H}^{H} \mathbf{H}+\sigma_{w}^{2} \mathbf{I}_{\mathcal{C}}$ with the aid of, for example, the ubiquitous Cholesky factorization [1]. Consequently, we can rewrite Eq.(3) as [6]:

$$
\begin{gathered}
\hat{\mathbf{s}}_{M L}=\arg \min _{\check{\mathbf{s}} \in M_{c}^{M}}\left(\check{\mathbf{s}}-\hat{\mathbf{x}}_{c}\right)^{H} \mathbf{U}^{H} \mathbf{U}\left(\check{\mathbf{s}}-\hat{\mathbf{x}}_{c}\right), \\
=\arg \min _{\check{\mathbf{s}} \in M_{c}^{M}} \sum_{i=1}^{M} \underbrace{u_{i i}^{2}\left[\check{s}_{i}-\hat{x}_{i}+\sum_{j=i+1}^{M} \frac{u_{i j}}{u_{i i}}\left(\check{s}_{j}-\hat{x}_{j}\right)\right]^{2}}_{\phi}
\end{gathered}
$$

3) Tree Search: All types of conventional SDs carry out the tree search based on the accumulated Partial Euclidean Distance (PED) evaluation corresponding to the term $\phi$ in Eq.(5). For the $K$-best SD [2], instead of expanding every node at each tree search level, we only retain a fixed number of $K$ nodes having the smallest accumulated PEDs. Hence, after the search reaches the tree leaf level, a candidate list $\mathcal{L}$ is generated, which contains $N_{\text {cand }}=K$ number of candidate solutions, which are then used for the extrinsic Log-Likelihoold-Ratio (LLR) calculation by the iterative SoftInput-Soft-Output (SISO) receiver according to [6]:

$$
\begin{gathered}
L_{E}\left(b_{k} \mid \mathbf{y}\right) \approx \frac{1}{2} \max _{\mathbf{b} \in \mathcal{L} \cap \mathbb{B}_{k,+1}}\left\{-\frac{1}{\sigma^{2}}\|\mathbf{y}-\mathbf{H} \mathbf{s}\|^{2}+\mathbf{b}_{[k]}^{T} \cdot \mathbf{L}_{A,[k]}\right\} \\
-\frac{1}{2} \max _{\mathbf{b} \in \mathcal{L} \cap \mathbb{B}_{k,-1}}\left\{-\frac{1}{\sigma^{2}}\|\mathbf{y}-\mathbf{H s}\|^{2}+\mathbf{b}_{[k]}^{T} \cdot \mathbf{L}_{A,[k]}\right\}
\end{gathered}
$$

\section{B. Center-Shifting Theory for SDs}

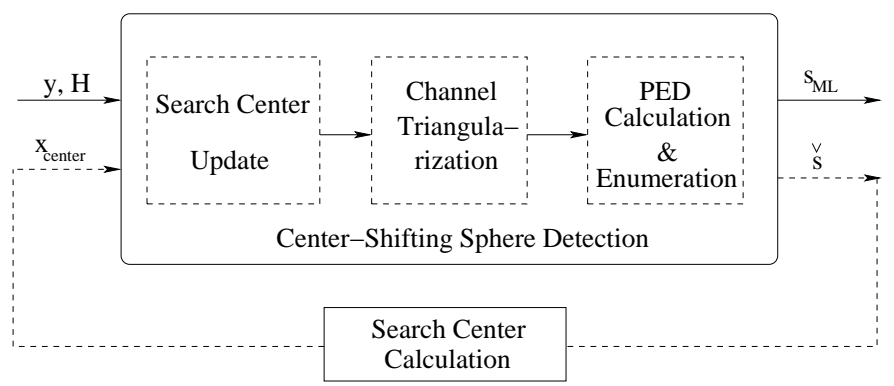

Fig. 1. The structure of the iterative SD using center-shifting scheme.

According to Eq.(3), when using sphere detection, the ML solution can be found by creating a reduced-size search-hypersphere centered around the MMSE solution by choosing an appropriate value for $K$. During our investigations, we realized that it would be desirable to set the SD's search center to a
MIMO signal constellation point, which may be expected to be closer to the real ML solution than the MMSE solution because this would allow us to reduce the SD's search space and hence its complexity. To some extent, extending the SD's search from a more accurate search center can be considered as a process of search-complexity reduction. Hence, it is plausible that the closer the search center is located to the real ML solution, the lower the computational complexity. More explicitly, the search can be carried out independently of the search center calculation. Thus, the search center can be obtained by more sophisticated detection schemes, not only by the conventional MMSE detection scheme. This observation turns the SD into a high-flexiblility detector, which can be readily combined with other well-established linear or non-linear detectors. As a result, the total computational complexity imposed by the $\mathrm{SD}$ is constituted by that of the detector, which provides the search center for the consecutive search operation of Figure 1. In other words, the affordable computational complexity can be flexibly split between the center calculation phase and the search phase of Figure 1, where the triangularization of the channel matrix $\mathbf{H}$ and the PED calculation previously detailed in Section III-A is portrayed explicitly. It is also plausible that an improved performance versus complexity trade-off emerges, if the search-center calculation is regularly updated, before further triangularization and PED calculation is carried out, as seen in Figure 1.

\section{SIC-MMSE-Aided Iterative Center-Shifting SD Assisted Receiver Architecture}

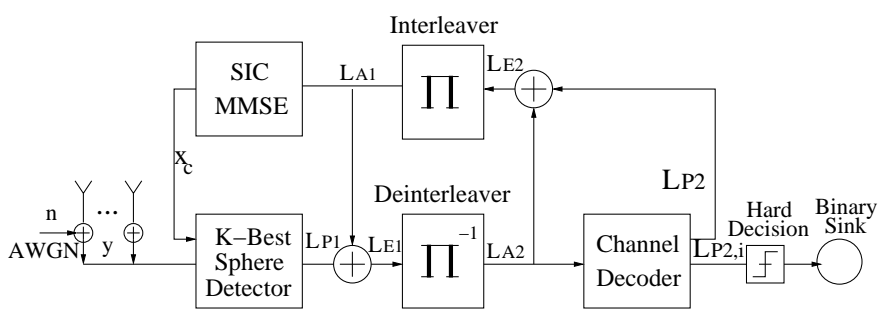

Fig. 2. Receiver Architecture of the SIC-MMSE-aided iterative centershifting $K$-best SD scheme.

1) Iterative Center-Shifting SD Assisted Receiver Architecture: Based on the observations provided in Section III-B, we can infer that the center-shifting scheme applied for the $\mathrm{SD}$ is expected to become significantly more powerful, if it is employed in the scenario of the iterative detection aided channel coded system of Figure 2. This is because the process of obtaining a more accurate search center is further aided by the channel decoder, which substantially contributes towards the total error-correction capability of the iterative receiver.

In Figure 2 the interleaver and deinterleaver pair seen at the receiver side divides the receiver into two parts, namely the inner MIMO detector and the outer channel decoder. Note that in Figure $2 L_{A}, L_{E}$ and $L_{D}$ denote the a priori, the extrinsic and the a posteriori LLRs, while the subscript ' 1 ' and ' 2 ' represent the bit LLRs associated with the inner detector and outer channel decoder respectively. It was detailed throughout [7] and [8] that the iterative exchange of extrinsic information between these serially concatenated receiver blocks results 
in substantial performance improvements. In this treatise we assume familiarity with the classic turbo detection principles [7,8]. Natually, the inner MIMO detector, namely the SD, has to be able to process the soft bit information provided by the soft-output channel decoder. On the other hand, the outer channel decoder also has to be able to process the soft reliability information provided by the soft-output inner SD. The resultant soft bit information is iteratively exchanged between the inner SD and the outer channel decoder.

In comparison to the conventional SD-aided iterative receiver of [6], the receiver proposed in Figure 2 has an additional functional block for calculating the search center $\mathbf{x}_{c}$ at specific iterations with the aid of MMSE Soft-InterferenceCancellation (SIC-MMSE) algorithm [9]. Hence, every time the search center $\mathbf{x}_{c}$ is updated, the $\mathrm{SD}$ is required to regenerate the candidate list [6], which is used to calculate the extrinsic LLRs delivered to the outer channel decoder.

\begin{tabular}{|c|c|c|c|c|}
\hline \multicolumn{5}{|c|}{ 4-QAM Symbol Alphabet $(i$ denotes $\sqrt{-1})$} \\
\hline$m$ & 1 & 2 & 3 & 4 \\
\hline$b_{m, 1} \quad b_{m, 2}$ & 00 & 01 & 10 & 11 \\
\hline$s_{m}$ & $(1+i) / \sqrt{2}$ & $(-1+i) / \sqrt{2}$ & $(1-i) / \sqrt{2}$ & $(-1-i) / \sqrt{2}$ \\
\hline
\end{tabular}

TABLE I

4-QAM SYMBOL ALPHABETS OVER THE COMPLEX NUMBERS

2) MMSE Soft-Interference-Cancellation Algorithm: In the context of the iterative receiver of Figure 2, given the $a$ priori LLRs provided by the FEC decoder, we can define the $m$ th transmit antenna's associated soft output symbol, more precisely, the mean of its soft output symbol as [9]:

$$
\bar{s}_{m}=E\left[s_{m}\right]=\sum_{q} s_{m}^{(q)} \cdot P\left[s_{m}=s_{m}^{(q)}\right],
$$

where $q$ is the number of points in the modulation constellation. Hence, we have $q=4$ for 4QAM or QPSK, while $s_{m}^{(q)}$ represents the $q$ th legitimate value of the symbol $s_{m}$. The symbol alphabet of the 4-QAM scheme is shown in Table I. Consequently, for 4-QAM, we arrive at:

$$
\begin{aligned}
s_{m} & =\left[\Re\left(s_{m}\right) ; \Im\left(s_{m}\right)\right], \\
& =\left[P\left[b_{m, 2}=-1\right] \cdot(+1)+P\left[b_{m, 2}=+1\right] \cdot(-1) ;\right. \\
& \left.P\left[b_{m, 1}=-1\right] \cdot(+1)+P\left[b_{m, 1}=+1\right] \cdot(-1)\right] / \sqrt{2},
\end{aligned}
$$

where $P\left[b_{k}= \pm 1\right]$ can be computed according to [7]:

$$
P\left[b_{k}=+1\right]=\frac{1}{1+e^{-L\left(b_{k}\right)}}
$$

and

$$
P\left[b_{k}=-1\right]=\frac{1}{1+e^{+L\left(b_{k}\right)}},
$$

respectively. On the other hand, we define the covariance of the symbol transmitted from the $m$ th antenna as [9] [10]:

$$
\begin{aligned}
v_{m} & =\operatorname{Cov}\left[s_{m}, s_{m}\right], \\
& =E\left[s_{m} s_{m}^{*}\right]-E\left[\bar{s}_{m}\right] E\left[\bar{s}_{m}^{*}\right], \\
& =1-\left|\bar{s}_{m}\right|^{2},
\end{aligned}
$$

for constant-modulus modulation schemes, such as BPSK, QPSK and 4-QAM.
The estimated symbol of the $m$ th transmit antenna generated by the MMSE algorithm can be expressed with the aid of the soft interference cancellation principle as [9]:

$$
\hat{s}_{m}=\bar{s}_{m}+v_{m} \mathbf{w}_{m}^{H}(\mathbf{y}-\mathbf{H} \overline{\mathbf{s}}),
$$

where the $m$ th column of the MMSE weight matrix $\mathbf{W}_{M M S E}$ can be expressed as [9]:

$$
\mathbf{w}_{m, M M S E}=\left(\mathbf{H V} \mathbf{H}^{H}+2 \sigma_{w}^{2} \mathbf{I}_{P}\right)^{-1} \mathbf{h}_{m}
$$

where $\mathbf{I}_{P}$ represents the $(P \times P)$-element identity matrix and $\mathbf{V}=\operatorname{diag}\left[v_{1}, v_{2}, \cdots, v_{M}\right]$.

3) Computational Complexity of the Iterative-CenterShifting-Aided K-Best SD: First of all, let us divide the complexity imposed by the $K$-best SD into two contributions, which are associated with the candidate-list generation (SD part) and the extrinsic LLR calculation (MAP part), respectively. Furthermore, we quantify the complexity of the list generation in terms of the number of PED evaluations corresponding to the term $\phi$ of Eq.(5). Hence, the list-generationrelated complexity can be approximated as:

$$
\mathcal{C}_{S D} \leq M \cdot \mathcal{M}_{C} \cdot K
$$

number of PED evaluations. On the other hand, the complexity imposed by the extrinsic LLR calculation is quantified in terms of the number of objective function (OF) evaluations, which corresponds to the two terms in Eq.(6). The approximation in Eq.(6) becomes an equality, when $\mathcal{L}$ represents the entire search space, constituted by $\mathcal{N}_{\text {cand }}=\mathcal{M}_{c}^{M}=2^{M \cdot B P S}$ number of $\mathrm{OF}$ evaluations, where $B P S$ is the number of bits per symbol. Hence, the complexity of the resultant exact MAP detector can be calculated as the total number of OF evalutions given by:

$$
\mathcal{C}_{M A P}=M \cdot B P S \cdot 2^{(M \cdot B P S)} .
$$

Clearly, the complexity grows exponentially with the product of the number of transmit antennas $M$ and the number of bits per symbol $B P S$. Let us consider an 8-transmit-antenna 4QAM SDM system as an example, which imposes an excess complexity of $\mathcal{C}_{M A P}=1,048,576$ OF evaluations.

Fortunately, the complexity may be significantly reduced by generating a list of candidates having a length of $\mathcal{N}_{\text {cand }}$ with the aid of the $K$-best SD, where we have $2^{M \cdot B P S} \geq \mathcal{N}_{\text {cand }} \geq$ 1 , since the corresponding complexity can be expressed as:

$$
\mathcal{C}_{M A P}=M \cdot B P S \cdot \mathcal{N}_{\text {cand }} .
$$

Consequently, the complexity has become linearly proportional to the length of the list $\mathcal{L}$. We will demonstrate with the aid of our forthcoming simulation results that the value of $\mathcal{N}_{\text {cand }}$ can be set to a small fraction of $2^{M \cdot B P S}$ with the aid of the proposed center-shifting scheme, especially when a high-throughput modulation scheme, e.g. 64QAM, and/or a large number of transmit antennas are employed by the system.

\section{Simulation Results And Discussion}

The benefits of applying the SIC-MMSE-aided centershifting scheme become more explicit, if we consider the EXtrinsic Information Transfer (EXIT) charts [11] obtained 


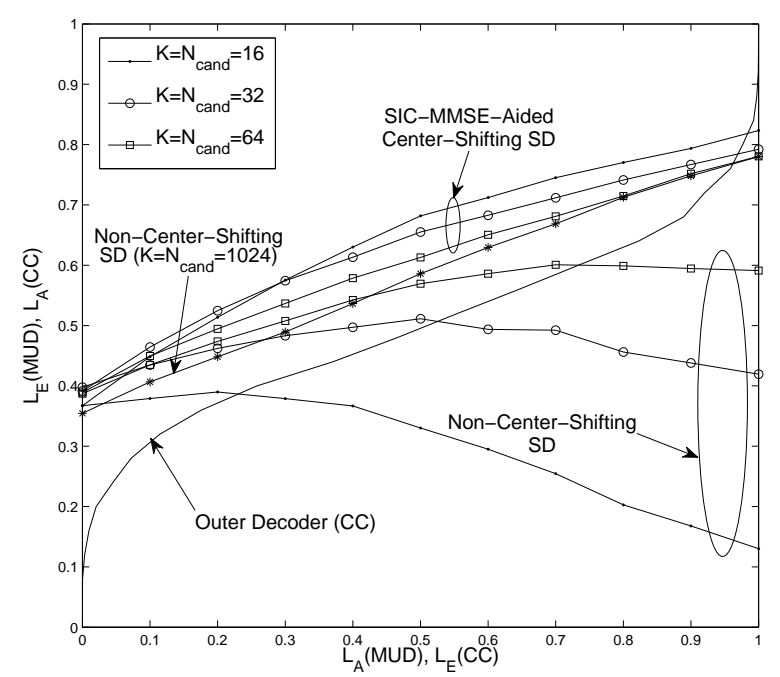

Fig. 3. EXIT chart comparison of SIC-MMSE-aided center-shifting and the non-center-shifting $K$-best SD iterative receiver in the scenario of an $(8 \times 4)$ element rank-deficient 4-QAM SDM/OFDM system at $\mathrm{SNR}=8 \mathrm{~dB}$. All other system parameters are listed in Table II.

\begin{tabular}{|c|c|}
\hline System Parameters & Choice \\
\hline System & $\overline{\text { SDM/OFDM }}$ \\
\hline No. of Sub-Carriers & 128 \\
\hline Modulation & 4-QAM \\
\hline No. of Transmit Antenna & 8 \\
\hline No. of Receive Antenna & 4 \\
\hline Block Length & 10240 \\
\hline CIR Model & $P\left(\tau_{k}\right)=\left[\begin{array}{lll}0.5 & 0.3 & 0.2\end{array}\right],(k=0,1,2)$ \\
\hline CIR Tap Fading & OFDM symbol invariant \\
\hline Channel Estimation & Ideal \\
\hline Detector/MAP & $K$-Best List-SD \\
\hline List Length $\mathcal{N}_{\text {cand }}$ & $=K$ \\
\hline \multirow{3}{*}{ Channel Encoder } & RSC $(2,1,3)$ \\
\hline & Generator Polynomials (6/13) \\
\hline & Code Termination (Off) \\
\hline No. of Iterations (Variable) & $\begin{array}{l}\text { Iterations terminate as soon as } \\
\text { the resultant trajectory line } \\
\text { reaches the convergence point }\end{array}$ \\
\hline
\end{tabular}

TABLE II

Summary OF System PARAMETERS FOR THE K-Best SD AIDED Coded SDM/SDMA OFDM SYSTEM

in the $(8 \times 4)$-element rank-deficient scenario of a 4-QAM SDM/OFDM system, as seen in Figure 3. Observe in Figure 3 that in the absence of our proposed center-shifting scheme the inner decoder's EXIT curve decayed upon increasing the $a$ priori information owing to the flawed information exchange between the inner and outer decoders, which was caused by the employment of an insufficiently large candidate list size $N_{\text {cand }}$ and the low number of candidates $K$ retained at each search level. The comparisons in Figure 3 indicate that this problem was effectively solved by the application of the SIC-MMSEaided center-shifting scheme. More explicitly, when using the SIC-MMSE scheme, the inner decoder's EXIT curve no longer decays, when the a priori mutual information (MI) increases, even when using a limited list size of $K=N_{\text {cand }}=16$ in this specific scenario. On the other hand, as shown in Figure 3, when $K$ and $N_{\text {cand }}$ are increased to 1024 for the $K$-best SD using no center-shifting, the resultant inner decoder's EXIT

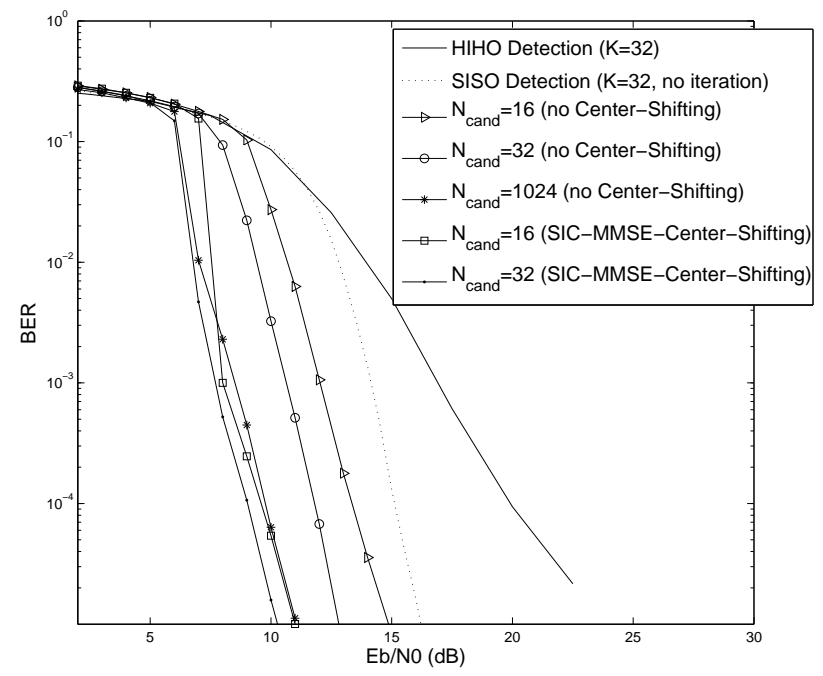

Fig. 4. BER performance improvement achieved by the SIC-MMSEaided iterative center-shifting $K$-best SD receiver in the scenario of an $(8 \times 4)$-element rank-deficient 4-QAM SDM/OFDM system. All other system parameters are listed in Table II.

curves increase. As expected, the EXIT curve corresponding to $K=N_{\text {cand }}=1024$ reaches a higher end point than that associated with low $K$ and $N_{\text {cand }}$ values. However, as a benefit of the SIC-MMSE center-shifting scheme, the EXIT curve of the inner decoder may arrive at an even higher end point, despite using significantly smaller $K$ and $N_{\text {cand }}$ values than that of the SD dispensing with center-shifting. Hence, we can infer from the above observations that the SIC-MMSE-aided receiver is capable of achieving a near-MAP BER performance conjunction with small values of $K$ and $N_{\text {cand }}$.

Our EXIT-chart based predictions were verified by the BER results of Figure 4, where a significant performance gain was achieved by the SIC-MMSE-aided center-shifting scheme. Specifically, as seen in Figure 4, the SIC-MMSE-aided iterative center-shifting $K$-best SD using $K=16$ is capable of approaching the performance of the iterative SD dispensing with center-shifting in conjunction with $K=1024$, at a BER of $10^{-5}$. Hence, both the associated memory requirements and the computational complexity imposed are substantially reduced. Explicitly, for a fixed value of $K$, such as for example $K=32$ and for the same target BER of $10^{-5}$, we can observe that the iterative gain over the non-iterative Soft-Input-SoftOutput (SISO) receiver was doubled to about $6 \mathrm{~dB}$ by the SICMMSE-aided center-shifting scheme, when compared to that achieved by the iterative SD dispensing with center-shifting.

Furthermore, with reference to Figure 5, the candidate list generation complexity of the SIC-MMSE center-shifting-aided receiver is well below that of the receiver using no centershifting for the SNR range spanning from $2 \mathrm{~dB}$ to $12 \mathrm{~dB}$. This statement is valid, if our aim is to achieve the near-MAP BER performance quantified in Figure 4, which can be attained by having $K=N_{\text {cand }}=1024$ for the system operating without the center-shifting scheme or by setting $K=N_{\text {cand }}=16$ in the presence of the center-shifting scheme. Actually, the number of PED-evaluations carried out per channel use by 


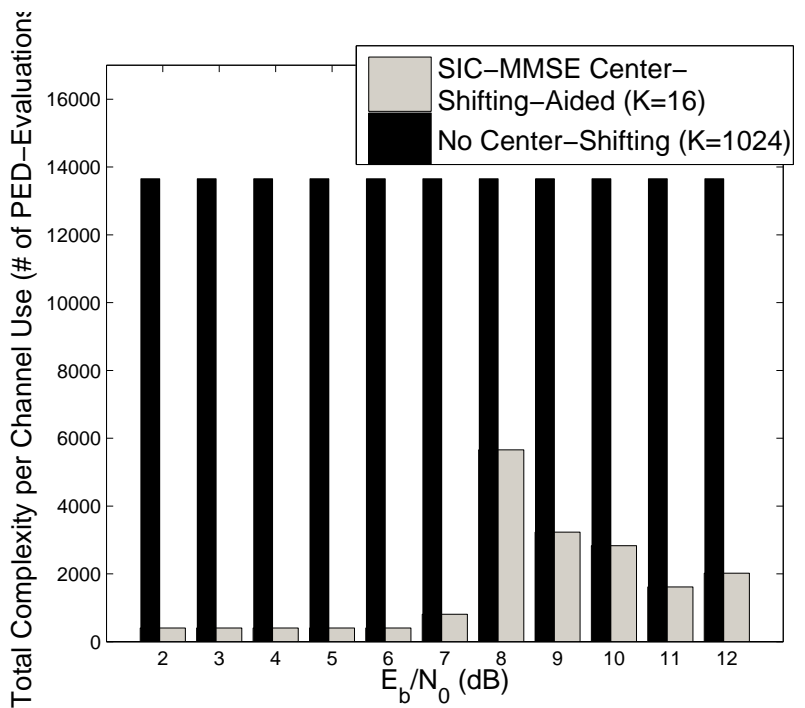

Fig. 5. Computational complexity histogram of the SIC-MMSE-aided iterative center-shifting $K$-best SD $(K=16)$ receiver in the scenario of an $(8 \times 4)$-element rank-deficient 4 -QAM SDM/OFDM system. All other system parameters are listed in Table II.

the system dispensing with the center-shifting scheme remains as high as 13,652, regardless of the SNR and the number of iterations. On the other hand, in the presence of the centershifting scheme, the candidate list has to be regenerated at each iteration, but nonetheless, the total complexity imposed is substantially reduced.

\begin{tabular}{|c|c|c|c|c|c|c|}
\hline \multicolumn{7}{|c|}{$\begin{array}{l}\text { Performance Gain \& Complexity Reduction Achieved by the SIC-MMSE } \\
\text { Scheme in }(8 \times 4) \text {-Element 4-QAM SDM/OFDM System (Target BER } 10^{-5} \text { ) }\end{array}$} \\
\hline $\begin{array}{l}\text { Center- } \\
\text { Shifting }\end{array}$ & $\begin{array}{l}N_{\text {cand }} \\
(K)\end{array}$ & Iter. & $\overline{\text { SNR }}$ & Memory & $\begin{array}{c}\text { SD } \\
\text { Compl. }\end{array}$ & $\begin{array}{l}\text { MAP } \\
\text { Compl. }\end{array}$ \\
\hline \multirow{5}{*}{ NONE } & 1024 & 3 & 10.5 & 8196 & 13652 & 49152 \\
\hline & 128 & 3 & 11.2 & 1024 & 2388 & 6144 \\
\hline & 64 & 2 & 12 & 512 & 1364 & 2048 \\
\hline & 32 & 2 & 12.8 & 256 & 724 & 1024 \\
\hline & 16 & 2 & 15 & 128 & 404 & 512 \\
\hline \multirow{3}{*}{ SIC-MMSE } & 64 & 3 & 10.2 & 512 & 4092 & 3072 \\
\hline & 32 & 3 & 10.2 & 256 & 2172 & 1536 \\
\hline & 16 & 3 & 11 & 128 & 1212 & 768 \\
\hline
\end{tabular}

TABLE III

Finally, we quantify the achievable performance gain and the complexity-reduction facilitated by the SIC-MMSE-aided center-shifting scheme in Table III, in comparison to the conventional SD-aided iterative receiver dispensing with the center-shifting scheme. Table III quantifies the computational complexity imposed by the SD section in terms of the total number of PED evaluations, and that associated with the MAP part in terms of the total number of OF evaluations corresponding to the two terms in Eq.(6) as discussed in Section III-C3. Thus, as explicitly indicated in Table III, in order to achieve a near-MAP performance, i.e. to achieve a BER of $10^{-5}$ at an SNR below $11 \mathrm{~dB}$ in the context of an $(8 \times 4)$-element rank-deficient SDM/OFDM system, we have to use at least $K=N_{\text {cand }}=1024$ for the SD-aided iterative receiver using no center-shifting. By contrast, as a benefit of the SIC-MMSE-aided center-shifting scheme, we can achieve the same goal by setting $K=N_{\text {cand }}=16$, while imposing a factor of 11 lower computational complexity than that associated with the list-generation part and imposing a factor of 64 lower computational efforts by the soft-bit information calculation of the SD receiver using no centershifting. A further additional performance gain of $0.8 \mathrm{~dB}$ can be obtained by setting $K=32$, at the cost of a modestly increased computational complexity. Furthermore, the memory requirements are also reduced by a factor of 64 with the aid of our center-shifting scheme.

\section{CONCLUSiON}

A novel center-shifting theory for SDs was proposed, which can be combined with any well-established linear or nonlinear detector. Our proposed SIC-MMSE-aided solution may enable the iterative center-shifting SD to achieve a nearMAP performance, despite imposing a significantly reduced memory requirements and complexity in comparison to the receiver dispensing with the center-shifting scheme. Specifically, the SIC-MMSE center-shifting scheme may enable the iterative receiver to achieve a near-MAP performance in the challenging scenario of an $(8 \times 4)$-element rank-deficient 4 QAM SDM/OFDM system. This near-MAP performance is achieved, despite imposing a reduced detection-candidate-listgeneration-related complexity, which is about an order of magnitude lower than that exhibited by the list-SD dispensing with the proposed center-shifting scheme. As a further benefit, the computational complexity associated with the extrinsic LLR calculation was reduced by a factor of about 64 . The associated memory requirements were also reduced by a factor of 64 .

\section{REFERENCES}

[1] E. Viterbo and J. Boutros, "A universal lattice code decoder for fading channels," IEEE Transactions on Information Theory, vol. 45, pp. 16391642, July 1999.

[2] K. Wong, C. Tsui, R. S. K. Cheng, and W. Mow, "A VLSI architecture of a k-best lattice decoding algorithm for MIMO channels," Circuits and Systems, 2002. ISCAS 2002. IEEE International Symposium on, vol. 3, pp. 273-276, May 2002.

[3] L. Hanzo, M. Munster, B. J. Choi, and T. Keller, OFDM and MC-CDMA for Broadband Multi-User Communications, WLANs and Broadcasting. IEEE Press, 2003.

[4] P. Vandenameele, L. V. D. Perre, M. G. E. Engels, B. Gyselinckx, and H. J. D. Man, "A combined OFDM/SDMA approach," IEEE Journal on Selected Areas in Communications, vol. 18, pp. 2312-2321, Nov. 2000.

[5] T. Cui and C. Tellambura, "An efficient generalized sphere decoder for rank-deficient MIMO systems," 2004. VTC2004-Fall. 2004 IEEE 60th Vehicular Technology Conference, vol. 5, pp. 3689-3693, Sept. 2004.

[6] B. M. Hochwald and S. ten Brink, "Achieving near-capacity on a multiple-antenna channel," IEEE Transactions on Communications, vol. 51, pp. 389-399, Mar. 2003.

[7] L. Hanzo, T. H. Liew, and B. L. Yeap, Turbo Coding, Turbo Equalisation and Space-Time Coding for Transmission over Fading Channels. IEEE Press, 2002.

[8] L. Hanzo, C. H. Wong, and M. S. Yee, Adaptive Wireless Transceivers. IEEE Press, 2002.

[9] M. Tuchler, A. C. Singer, and R. Koetter, "Minimum mean squared error equalization using a priori information," Signal Processing, IEEE Transactions on Acoustics, Speech, and Signal Processing, vol. 50, pp. 673-683, Mar. 2002.

[10] L. Xu, S. Tan, S. Chen, and L. Hanzo, "Iterative minimum bit error rate multiuser detection in multiple antenna aided OFDM," 2006. WCNC 2006. IEEE Wireless Communications and Networking Conference, vol. 3, pp. 1603-1607, Apr. 2006.

[11] S. ten Brink, "Convergence behavior of iteratively decoded parallel concatenated codes," IEEE Transactions on Communications, vol. 49, pp. 1727-1737, Oct. 2001. 